\title{
Características clínicas y manifestaciones infecciosas en pacientes con Enfermedad Granulomatosa Crónica (EGC) en Paraguay
}

\author{
Clinical characteristics and infectious manifestations in \\ patients with Chronic Granulomatous Disease (CGD) in \\ Paraguay
}

\author{
Celia Martínez de Cuellar ${ }^{1,2}$, Dolores Lovera ${ }^{1}$, Sara Amarilla', Luis Gatti', Silvio Apodaca ${ }^{1}$, \\ Diana Sanabria ${ }^{2}$, Antonio Arbo ${ }^{1,2}$
}

\section{RESUMEN}

Introducción: La enfermedad granulomatosa crónica (EGC) es causada por un defecto en una de las subunidades de nicotinamida fosfato (NADPH) oxidasa, produciendo en los fagocitos una falla en la producción del superóxido. Como resultado, los pacientes presentan infecciones bacterianas y fúngicas recurrentes. Objetivos: analizar las características clínicas, manifestaciones infecciosas, los microrganismos aislados, así como la evolución de los pacientes que fueron diagnosticados en el Instituto de Medicina Tropical desde en el periodo 1991 2017. Materiales y Métodos: fueron incluidos pacientes con diagnóstico de EGC. Se revisaron los expedientes clínicos de pacientes con EGC hospitalizados, se determinaron las características clínico-demográficas, incluyendo la edad del inicio de las manifestaciones clínicas, edad de diagnóstico, antecedentes de consanguinidad en la familia, historia familiar de cuadros similares, manifestaciones clínicas, frecuencia de cuadros infecciosos, complicaciones asociadas a la vacuna BCG, días de hospitalización y evolución de la enfermedad. Resultados: se identificaron 11 pacientes (8 varones y 3 mujeres). Todos los pacientes pertenecían a 8 familias, se identificaron 2 familias con más de un miembro afectado (1 familia con 2 hermanas y otra con 2 primos y una tía afectados). Dos de los 11 pacientes tenían antecedentes de familiares con muerte prematura dentro de las 3 generaciones. La edad media de inicio de síntomas fue de $14.5 \pm 10,3$ meses y al momento del diagnóstico de $32.4 \pm 25,06$ meses. En un paciente se identificó antecedentes de consanguinidad, aunque todos recibieron

\section{ABSTRACT}

Introduction: Chronic granulomatous disease (CGD) is caused by a defect in one of the subunits of nicotinamide phosphate (NADPH) oxidase, producing a failure in the production of superoxide in phagocytes. As a result, patients have recurrent bacterial and fungal infections. Objectives: to analyze the clinical characteristics, infectious manifestations, the isolated microorganisms, as well as the evolution of patients that were diagnosed at the Institute of Tropical Medicine from 1991 to 2017. Materials and Methods: patients with a diagnosis of CGD were included. Clinical records of hospitalized CGD patients were reviewed, clinical-demographic characteristics were determined, including the age of onset of clinical manifestations, age of diagnosis, family history of consanguinity, family history of similar conditions, clinical manifestations, frequency of infectious diseases, complications associated with the BCG vaccine, hospitalization days and disease evolution. Results: 11 patients were identified ( 8 men and 3 women). All the patients belonged to 8 families, 2 families were identified who had more than one affected member ( 1 family with 2 sisters and another with 2 affected cousins and one aunt). Two of the 11 patients had a family history of premature death within 3 generations. The mean age of symptom onset was $14.5 \pm 10.3$ months and age at the time of diagnosis was $32.4 \pm 25.06$ months. In one patient, a history of consanguinity was identified; although all patients received the $B C G$ vaccine, none presented a history of BCG adenitis. The mean number of infectious cases that required hospitalization was $3 \pm 1.7$. During the study

\footnotetext{
${ }^{1}$ Instituto de Medicina Tropical, Servicio de Pediatría. Asunción, Paraguay.

${ }^{2}$ Universidad Nacional de Asunción. San Lorenzo, Paraguay.

Correspondencia: Celia Martínez de Cuellar Correo: zhelia.martinez@yahoo.com

Conflicto de Interés: Los autores declaran no poseer conflicto de interés

Recibido: 7/05/2018 Aceptado: 24/07/2018

DOI: https://doi.org/10.31698/ped.45022018004
} 
la vacuna $B C G$, ninguno presento historia de becegeitis. La media de cuadros infecciosos que requirieron hospitalización fue de $3 \pm 1,7$. Durante el periodo de estudio, los pacientes presentaron 38 cuadros infecciosos. Las infecciones pulmonares fueron las más frecuentemente observadas $42,1 \%(16 / 38)$, incluyendo las neumonías (9/38), absceso pulmonar (1/38), tuberculosis pulmonar $(1 / 38)$ y bronquiolitis (14/38). Otros cuadros infecciosos que requirieron hospitalización fueron las sepsis 18,4\% (7/38), uno de ellos con endocarditis, adenitis $13,2 \%(5 / 38)$, infecciones de piel y partes blandas $13,2 \%$ (5/38), meningitis bacteriana y osteomielitis 2,6\% (1/38), respectivamente. En 19 de los cuadros infecciosos se aisló el microrganismo causante, siendo el más frecuente el $S$. aureus $(\mathrm{n}=9)$, pero en 10 casos se aisló un microrganismo inusual [Aspergillus $(\mathrm{n}=4)$, Chromobacterium violaceum, Burkholderia cepacia, Enterobacter cloacae, Serratia marcescens, Mycobacterium tuberculosis (uno en cada caso)]. El tiempo medio de hospitalización fue de 23,3 314,1 días y la mortalidad acumulada de $3 / 11$. Conclusiones: Coincidente con otras series la mayoría de los casos de EGC, en Paraguay, se observó en varones, y la edad de inicio de síntomas fue antes de los 2 años de edad. El diagnóstico de la enfermedad fue relativamente precoz. El sitio infeccioso más frecuente fue el pulmonar, siendo el microorganismo más frecuentemente aislado el S. aureus, aunque se aislaron microorganismos inusuales en una proporción significativa. Es fundamental considerar esta ID en pacientes con cuadros infecciosos de evolución tórpida y por microorganismos inusuales.

Palabras claves: Enfermedad granulomatosa crónica, inmunodeficiencias Primarias.

\section{INTRODUCCIÓN}

La enfermedad granulomatosa crónica (EGC) es una inmunodeficiencia primaria, descripta por primera vez en el año 1950, con una incidencia de 1 cada 200 000 a 250000 en recién nacidos vivos ${ }^{(1,2,3)}$.

La EGC no se desencadena en las células fagocíticasprincipalmente neutrófilos y macrófagos- durante el evento fagocítico el estallido respiratorio, mecanismo molecular por el cual se generan en el citoplasma celular peróxido de hidrógeno, superóxido y otros compuestos activados del $\mathrm{O}_{2}$, por una deficiencia en la actividad de la nicotinamida adenina dinucleótido fosfato-oxidasa (NADPH oxidasa) ${ }^{(4-7)}$. La función microbicida defectuosa de las células fagocíticas tiene finalmente como consecuencia que las bacterias y los hongos a pesar de ser fagocitados no son period, patients presented with 38 infectious conditions. Pulmonary infections were the most frequently observed $42.1 \%$ (16/38), including pneumonia (9/38), lung abscess $(1 / 38)$, pulmonary tuberculosis $(1 / 38)$ and bronchiolitis $(14 / 38)$. Other infectious conditions that required hospitalization were sepsis $18.4 \%(7 / 38)$, one of them with endocarditis, adenitis $13.2 \%(5 / 38)$, skin and soft tissue infections $13.2 \%(5 / 38)$, bacterial meningitis and osteomyelitis $2.6 \%(1 / 38)$, respectively. In 19 of the infectious conditions, the causative microorganism was isolated, the most frequent being $S$. aureus $(n=9)$, but in 10 cases an unusual microorganism was isolated [Aspergillus $(\mathrm{n}=4)$, Chromobacterium violaceum, Burkholderia cepacia, Enterobacter cloacae, Serratia marcescens, Mycobacterium tuberculosis (one in each case)]. The mean hospitalization time was $23.3 \pm 14.1$ days and the accumulated mortality of $3 / 11$. Conclusions: Coinciding with other series, most cases of CGD in Paraguay were observed in males, and the age of onset of symptoms was before 2 years of age.The diagnosis of the disease was made relatively early. The most frequent infectious site were the lungs, with the microorganism most frequently isolated being $S$. aureus, although unusual microorganisms were isolated in a significant number of cases. It is essential to consider this diagnosis in patients with recurrent bouts of infectious conditions and caused by unusual microorganisms.

Keywords: Chronic granulomatous disease, primary immunodeficiencies.

destruidos $^{(4,5)}$. Este defecto además impide la eliminación de restos celulares aumentando la inflamación favoreciendo la formación de granulomas, hallazgo anatomoatológico que ha dado el nombre a la enfermedad ${ }^{(4-7)}$.

Esta rara enfermedad, se hereda principalmente en forma recesiva ligada al cromosoma X en $65 \%$ a $67 \%$ de los casos, y resulta con una mayor incidencia en los hombres que en las mujeres. En una menor proporción se hereda en forma autosómica recesiva. La EGC, ligada al cromosoma $X$ en general es más severa y se inicia dentro de los primeros años de vida, mientras que las formas autosómicas recesivas son menos severas y el cuadro clínico en general se inicia más tardíamente entre los 5 y 7 años ${ }^{(2,8,9)}$. La 
tasa de mortalidad de pacientes recesivos ligados a $X$ también es más alta que la de pacientes autosómicos recesivos $^{(2,3)}$.

Los pacientes con esta enfermedad son susceptibles a desarrollar infecciones severas en los primeros años de vida, que pueden afectar cualquier a órgano, sin embargo, los pulmones, la piel y tejido celular subcutáneo, tracto gastrointestinal, ganglios linfáticos, hígado y huesos son los sitios de infección más frecuentes ${ }^{(2)}$. Las infecciones en el paciente con EGC típicamente son causadas por bacterias catalasa positiva y hongos. Los microrganismos más comunes son Aspergillus sp, Staphylococcus aureus, Burkholderia cepacia, Serratia marcescens y Nocardia. El aislamiento de deteminados microorganismos en procesos infecciosos como Chromobacterium violaceum, Francisella philomiragia, Granulibacter bethesdensis, Acidomonas methanolica y Methylobacterium lusitanum sugieren la EGC como un trastorno subyacente ${ }^{(3,10-16)}$.

El diagnóstico de esta enfermedad se realiza confirmando la alteración del estallido respiratorio en los neutrófilos del paciente. Los métodos de diagnóstico más utilizados son el ensayo de citometría de flujo con dihidrorodamina y la prueba de colorante con nitroazul de tetrazolio. Actualmente la prueba genética es el método de diagnóstico más preciso disponible ${ }^{(3)}$.

En el año 1991 se realizaron los primeros diagnósticos de EGC en nuestro país, los cuales fueron comunicados en el año $1995^{(17)}$. En el presente reporte, se analizan las características clínicas, manifestaciones infecciosas, los microrganismos aislados, así como la evolución de los pacientes que fueron diagnosticados en el Instituto de Medicina Tropical desde en el periodo 1991-2017.

\section{MATERIALES Y MÉTODOS}

Objetivos: Analizar las características clínicas, manifestaciones infecciosas, los microrganismos aislados de los pacientes que fueron diagnosticados en el Instituto de Medicina Tropical, en el periodo 1991-2017.

Diseño: El presente estudio ha sido observacional descriptivo, retrospectivo de corte transversal.
Población: Fueron incluidos pacientes con diagnóstico de enfermedad granulomatosa crónica (EGC), confirmado por la prueba del nitro azul de tetrazolio (NBT), dihidrorodamina (DHR) y/o la identificación genética del defecto molecular, con edades comprendidas entre 0 a 15 años, que estuvieron hospitalizados en el Instituto de Medicina Tropical o centros asistenciales de salud tanto públicos como privados del país, y registrados en el Centro Nacional de Referencia de Inmunodeficiencias Primarias de la Infancia, Instituto de Medicina Tropical - Instituto de Investigaciones en Ciencias de la Salud, en el periodo enero de 1991 y diciembre de 2017.

Se revisaron los registros médicos de cada paciente para recolección de datos demográficos, clínicos y de laboratorio. Fueron evaluadas las siguientes variables: sexo, edad de inicio de síntomas, edad al diagnóstico, historia familiar de consanguinidad, muerte temprana de miembros de la familia, manifestación clínica, frecuencia de las infecciones, días de hospitalización, complicaciones por la vacuna BCG, microorganismos aislados, óbitos registrados.

\section{Criterios de inclusión:}

- Pacientes con infecciones a repetición con diagnóstico confirmado de EGC, por la prueba del nitro azul de tetrazolio (NBT) o la determinación de la dihidrorodamina (DHR).

\section{Criterios de exclusión:}

- Pacientes con infecciones a repetición con sospecha de EGC.

Aspectos éticos: El trabajo fue aprobado por el Comité de ética del IMT. Se mantuvo en todo momento el anonimato y la confidencialidad de la información recolectada. Este estudio no significó riesgo adicional alguno para los pacientes evaluados, debido a que los datos se obtuvieron a partir de una revisión retrospectiva de las fichas clínicas.

Análisis estadístico: Los datos recolectados fueron asentados en una hoja de cálculo de Microsoft Excel y posteriormente analizados con el programa estadístico SPSS versión 11.5 para Windows. Se empleó proporciones y porcentajes para describir variables categóricas, mientras que las cuantitativas 
fueron resumidas utilizando medias y desviación estándar.

\section{RESULTADOS}

Durante el periodo de estudio se identificaron 11 pacientes; 8 de ellos fueron del sexo masculino. La edad media de inicio de síntomas fue de $14.5 \pm 10,3$ meses y la edad media al momento del diagnóstico fue de 32.4 $\pm 25,06$ meses (Tabla 1). En la mayoría de los pacientes, los cuadros infecciosos se iniciaron entre los 1 y 5 años; sin embargo 4 pacientes iniciaron síntomas antes del año de edad (Tabla 2).

Ningún paciente tuvo antecedentes de consanguinidad ni historia de becegeitis. Todos los pacientes fueron identificados en 8 familias, un paciente refirió antecedentes de consanguinidad. Se observó que 2 familias tenían más de un miembro afectado (una familia con 2 hermanas afectadas y otra con 2 primos y una tía). Por otro lado, 2/11 tenían antecedentes de muertes prematuras por cuadros infecciosos en las 3 últimas generaciones.

Tabla 1. Características demográficas de los pacientes con EGC.

\begin{tabular}{lc}
\hline Datos demográficos & $\mathbf{n = 1 1}$ \\
\hline Edad (meses \pm DE) & $32.4 \pm 25,06$ \\
Edad de inicio de síntomas & $14.5 \pm 10,3$ \\
Sexo M/F & $8 / 3$ \\
Antecedentes de consanguinidad & 1 \\
Familias con más de un paciente & 2 \\
Familias con antecedentes de muertes prematuras & 2 \\
Becegeitis & 0 \\
\hline * Casos hospitalizados en el Institutito de Medicina Tropical, 2018 &
\end{tabular}

Tabla 2. Edad de inicio de síntomas y de diagnóstico.

\begin{tabular}{ccc}
\hline Grupo de edad & Edad de inicio de síntomas & Edad de Diagnóstico \\
\hline$<1$ & 4 & 0 \\
1 a 5 & 6 & 10 \\
$>5$ & 1 & 1 \\
\hline * Casos hospitalizados en el Institutito de Medicina Tropical, 2018 &
\end{tabular}

El diagnóstico de EGC se hizo en todos los pacientes por el test de NBT y/o DHR; en 4 pacientes (36\%) además se realizó la caracterización molecular del defecto genético, identificándose en 2 pacientes de sexo masculino un defecto de la glicoproteína de membrana gp91phox y en 2 pacientes de sexo femenino (hermanas) defecto de la proteína citosólica p47phox.
Los 11 pacientes presentaron 38 cuadros infecciosos. Las infecciones más frecuentes fueron las infecciones pulmonares representando el 42,1\% $(16 / 38)$ de todas las infecciones. Otros cuadros infecciosos frecuentes fueron sepsis que representó el 18,4\% (7/38) de los casos, adenitis/adenoflemón el $13,2 \%(5 / 38)$ e infecciones de piel y partes blandas el $13,2 \%(5 / 38)$ (Tabla 3$)$.

Tabla 3. Cuadros infecciosos en pacientes con EGC.

\begin{tabular}{lcc}
\hline Cuadros Infecciosos & $\mathbf{n = 3 8}$ & $\mathbf{\%}$ \\
\hline Infecciones pulmonares & 16 & 42,1 \\
Neumonía & 14 & 36,8 \\
Absceso pulmonar & 1 & 2,6 \\
Tuberculosis & 1 & 2,6 \\
\hline Bronquiolitis & 1 & 2,6 \\
Sepsis & 7 & 18,4 \\
Adenitis/Adenoflemón & 5 & 13,2 \\
Infecciones de piel y partes blandas & 5 & 13,2 \\
Otitis media aguda & 1 & 2,6 \\
Meningitis bacteriana & 1 & 2,6 \\
Osteomielitis & 1 & 2,6 \\
Gastroenteritis & 1 & 2,6 \\
Endocarditis & 1 & 2,6 \\
\hline * Casos hospitalizados en el Institutito de Medicina Tropical, 2018
\end{tabular}

Se obtuvo aislamiento del germen causal en 19 eventos infecciosos (Figura 1). S. aureus, representó el microogranismo mas frecuentemente aislado (9/19) en nuestra serie de pacientes con EGC. En diez casos se aislaron microorganismos inusuales; entre ellos Aspergillus (aislado en 4 pacientes), Chromobacterium violaceum (en 2 pacientes) y otros microorganismos como Burkholderia cepacia, Enterobacter cloacae, Serratia marcescens, Mycobacterium tuberculosis (un caso cada uno).

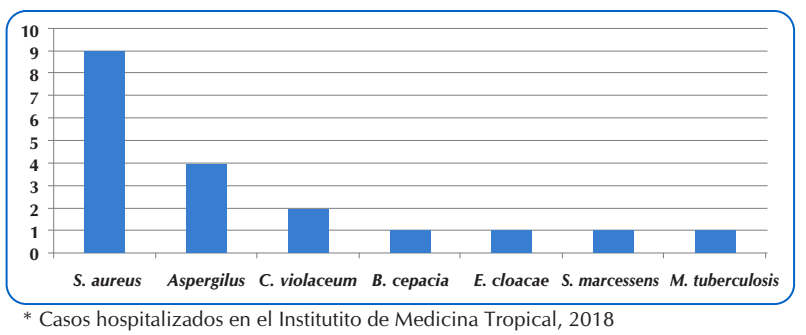

Figura 1. Microorganismos aislados en cuadros infecciosos de pacientes con EGC.

La media de cuadros infecciosos que requirieron hospitalización fue de $3 \pm 1,7$. El tiempo medio de hospitalización fue de $23,3 \pm 14,1$ días. Se identificó la presencia de granulomas en 1 paciente, cuya localización fue sub-endocárdica (Tabla 4). 
Una vez realizado el diagnóstico en todos los pacientes se indicó profilaxis con trimetropinsulfametoxazol e itraconazol. Ningún paciente recibió tratamiento definitivo con trasplante de médula ósea o ingeniería genética. Sin embargo, el $45 \%$ de los pacientes presentó cuadros infecciosos, aun cuando recibieron profilaxis antimicrobiana y antimicótica, el 79\% (30/38) de los cuadros infecciosos ocurrió antes del inicio de la quimioprofilaxis. La mortalidad acumulada fue del $27 \%$ (3/11). Al momento actual, la media de las edades de los pacientes sobrevivientes es de 14,8 \pm 13,2 años.

Tabla 4. Evolución de los pacientes hospitalizados con EGC.

\begin{tabular}{lc}
\hline Evolución & $\mathbf{n = 1 1}$ \\
$\mathrm{N}^{\circ}$ de hospitalizaciones $\pm \mathrm{DE}$ & $3 \pm 1,7$ \\
\hline Media del tiempo de hospitalizaciones (días $\pm \mathrm{DE})$ & $23,3 \pm 14,1$ \\
\hline Fallecidos N $(\%)$ & 3 \\
\hline * Casos hospitalizados en el Institutito de Medicina Tropical, 2018 &
\end{tabular}

\section{DISCUSIÓN}

La EGC se describió inicialmente como "una enfermedad granulomatosa mortal de la infancia". Históricamente, la mayoría de los pacientes con CGD moría en los primeros 10 años de edad ${ }^{(18)}$. Sin embargo, una mayor conciencia de la enfermedad y los avances en el diagnóstico ha llevado a una mejoría notable en la esperanza de vida. Antes de la introducción de antifúngicos orales, Winkelstein et al. refirieron una tasa de mortalidad del $5 \%$ por año para EGC ligada a $X$ y del $2 \%$ por año para CGD autosómica recesiva ${ }^{(2)}$.

La EGC, ligada al cromosoma $X$ en general es más severa y empieza dentro de los primeros años de vida, mientras que las formas autosómicas recesivas son menos severas y el cuadro clínico en general se inicia más tardíamente entre los 5 y 7 años ${ }^{(2,8,9)}$. La tasa de mortalidad de pacientes recesivos ligados a $X$ también es más alta que la de pacientes autosómicos recesivos $^{(2,3)}$.

Estudios más recientes informan una tasa de supervivencia de aproximadamente $90 \%$ a los 10 años de edad, que se ha atribuido a un mejor reconocimiento y diagnóstico temprano que condujo a terapias más tempranas, incluida una profilaxis antimicrobiana más eficaz, el uso de suplementos de interferón gamma (IFN- $\gamma$ ) para la profilaxis de infección y el uso de trasplante de células madre hematopoyéticas (HCT) ${ }^{(19)}$. Sin embargo, la mediana de la edad de la muerte se mantiene alrededor de 30-40 años, y los pacientes tienden a estar cada vez más debilitados con la mala calidad de vida del avance de la edad ${ }^{(3,20,21)}$.

En el presente artículo, se reportan las características demográficas y clínicas de los pacientes con Enfermedad Granulomatosa Crónica atendidos en el Centro Nacional de Referencia de Inmunodeficiencias del Paraguay. La enfermedad granulomatosa crónica es una rara enfermedad hereditaria, cuya prevalencia en Paraguay, en el año 2006, era de 0,26/100.000 habitantes ${ }^{(1)}$. Otras series reportan una prevalencia de 1 por cada 120.000 a 250.000 habitantes $^{(23-26)}$.

El primer caso de Enfermedad Granulomatosa Crónica fue reportado en el Paraguay en el año 1992 y durante los siguientes 25 años se ha realizado la búsqueda intensiva de Inmunodeficiencias Primarias de la Infancia en aquellos pacientes con infecciones a repetición o aquellos que presentaron infecciones por microrganismos inusuales, identificándose un total de 11 pacientes con EGC ${ }^{(17,22)}$.

En este reporte se puede observar que los pacientes con EGC presentaron una amplia variedad de cuadros infecciosos, y las causas más frecuentes fueron las infecciones bacterianas; principalmente del tracto respiratorio, seguidas de infecciones de ganglios linfáticos y la piel. Borges de OliveiraJunior y colaboradores refieren 71 casos de EGC en países de Latinoamérica, donde los cuadros infecciosos más frecuentes fueron los pulmonares, ganglionares y cutáneos (27) principalmente por bacterias y hongos. La serie de casos publicada por Winkelstein JA y col. informa que las neumonías fueron las infecciones más frecuentes, observándose en el $79 \%$ de los pacientes, donde el microrganismo más frecuentemente aislado fue el Aspergillus (2). Varios autores describieron casos de neumonía por Aspergillus sp asociada con la exposición ambiental de grandes cantidades de esporas fúngicas, que resulta ser la manifestación inicial de la enfermedad granulomatosa crónica en adolescentes y adultos jóvenes ${ }^{(6,25)}$. 
Solo en el 50\% (19/38) de los cuadros infecciosos se obtuvo aislamiento del microrganismo causante, y fueron aislados frecuentemente microrganismos inusuales como Chromobacterium violaceum, Burkholderia cepacia, Enterobacter cloacae, Serratia marcescens, Mycobacterium tuberculosis. Wilkestein JA y col. describieron el aislamiento de microrganismos inusuales con mayor frecuencia que en nuestra serie (70\% de los casos). Asimismo, Martire B y col refieren con mayor frecuencia los microrganismos inusuales (62\% de los casos).

Las infecciones producidas por micobacterias, en general son consideradas como parte del cuadro clínico en los reportes de Europa y EEUU ${ }^{(2,3,8,20)}$. Así mismo, las mismas son comunes en pacientes en otras áreas geográficas del mundo, incluyendo Latinoamérica, donde se ha observado un incremento de cuadros infecciosos producidos por este microorganismo, especialmente en países donde la Tuberculosis es endémica y donde la vacunación con BCG se encuentra dentro del esquema rutinario de vacunaciones. Oliveira Junior et al, mencionaron que el 53,5\% de sus pacientes presentaron Becegeitis ${ }^{(26)}$, lo cual no fue observado en ningún paciente de nuestra serie.

En nuestra serie, el intervalo de tiempo entre el inicio del cuadro clínico (media 14,5 meses), y el diagnóstico de la enfermedad, (media 32,4 meses), resultó inferior al reportado en la serie de casos de Latinoamérica, donde, las medias de inicio de los síntomas y la edad de diagnóstico fue de 23,9 meses y 52,7 meses, respectivamente ${ }^{(26)}$.

La enfermedad al igual que en otras series fue más frecuente en el sexo masculino. Así, en la serie italiana se refiere un predominio del $97 \%$ del sexo masculino; mientras que la serie Latinoamérica y el reporte de Winkelstein et al, mencionan un predominio de $82 \%$ y $78 \%$, respectivamente ${ }^{(8,20,26)}$

El antecedente de historia familiar de EGC se reporta en las diferentes series revisadas, al igual que lo reportado en nuestro estudio, donde observamos que los 11 casos provenían de 8 familias ${ }^{(8,20)}$. En nuestra serie observamos sólo un caso de antecedentes de cosanguinidad.

Una vez hecho el diagnóstico, todos los pacientes recibieron quimioprofilaxis antibiótica y antimicótica y aunque ningún paciente recibió tratamiento definitivo con trasplante de médula ósea o ingeniería genética, la mortalidad fue del $27 \%$. Una serie mexicana, informó una mortalidad superior a la reportado en este estudio (33\% entre 27 pacientes con EGC) ${ }^{(23)}$. Otros estudios, como el Martire B y col, en su estudio multicéntrico expresaron una mortalidad menor (13\%) y el grupo Latinoamericano del de Inmunodeficiencias Primarias no mencionó mortalidad ${ }^{(8,27)}$.

La causa más común de muerte reportada en las series de pacientes con EGC fue la neumonía y/o sepsis por Aspergillus o B. cepacia complex ${ }^{(2,21)}$. En nuestro estudio, la muerte de los pacientes se produjo durante cuadros infecciosos en 2 casos, y 1 caso debido a un cuadro infeccioso y presencia de granuloma, pero, en ninguno de estos casos se obtuvo aislamiento del microorganismo causante. Asimismo, Martire B y col. refieren que las infecciones producidas por hongos, no así las bacterianas son determinantes de la sobrevivencia y constituyen la principal causa de morbilidad y mortalidad por EGC ${ }^{(8)}$.

\section{CONCLUSIÓN}

En conclusión, coincidente con otras series, en Paraguay la mayoría de los casos de EGC se observó en varones, y la edad de inicio de síntomas fue antes de los 2 años de edad. El sitio infeccioso más frecuente fue pulmonar, el microorganismo más frecuentemente aislado fue el $S$. aureus, aunque se aislaron microorganismos inusuales en una proporción significativa. Es fundamental considerar esta inmunodeficiencia en pacientes con cuadros infecciosos de evolución tórpida y por microorganismos inusuales. 


\section{REFERENCIAS}

1. Leiva LE, Zelazco M, Oleastro M, Carneiro-Sampaio M, Condino-Neto A, Tavares Costa-Carvalho B, et al. Primary Immunode?ciency Diseases in Latin America: The Second Report of the LAGID1 Registry. Journal of Clinical Immunology. 2007;27(1):101-8.

2. Winkelstein JA, Marino MC, Johnston RB Jr, Boyle J, Curnutte J, Gallin JI, et al. Chronic granulomatous disease. Report on a national registry of 368 patients. Medicine (Baltimore), 2000; 79(3): 155-69.

3. van den Berg JM, van Koppen E, Ahlin A, Belohradsky $\mathrm{BH}$, Bernatowska E, Corbeel L, et al. Chronic granulomatous disease: the european experience. PLoS ONE. 2009; 4(4):e5234.

4. Geha RS, Notarangelo LD, Casanova JL, Chapel H, Conley ME, Fischer A, et al. Primary immunodeficiency diseases: an update from the International Union of Immunological Societies Primary Immunodeficiency Diseases Classification Committee. J Allergy Clin Immunol. 2007; 120: 776-794.

5. Nabavi M, Arshi S, Bemanian MH, Aghamohammadi A, Mansouri D, Hedayat M, et al. Long-term follow-up of ninety eight Iranian patients with primary immune deficiency in a single tertiary centre. Allergol Immunopathol. 2016; 44:322-330.

6. Segal BH, Leto TL, Gallin JI, Malech HL, Holland SM. Genetic, biochemical, and clinical features of chronic granulomatous disease. Medicine (Baltimore). 2000; 79(3):170-200.

7. Segal BH, Veys P, Malech H, Cowan MJ. Chronic granulomatous disease: lessons from a raredisorder. Biology of Blood and Marrow Transplantation. 2011; 17(1)Supplement:S123-S131.

8. Martire B, Rondelli R, Soresina A, Pignata C, Broccoletti $\mathrm{T}$, Finocchi A, et al. Clinical features, long-term follow up and outcome of a large cohort of patients with chronic granulomatous disease: An Italian multicenter study. Clin Immunol, 2008; 126:155-64.

9. Soler-Palacín P, Margareto C, Llobet P, Asensio O, Hernández M, Caragol I, et al: Chronic granulomatous disease in pediatric patients: 25 years of experience. Allergol Immunopathol (Madr), 2007; 35: 83-89.

10. Sirinavin S, Techasaensiri C, Benjaponpitak S, Pornkul $\mathrm{R}$, Vorachit, M. Invasive Chromobacterium violaceum infection in children: case report and review. The Pediatric Infectious Disease Journal. 2005; 24(6):559-561.
11. Mailman TL, Schmidt MH. Francisella philomiragia adenitis and pulmonary nodules in a child with chronic granulomatous disease. Canadian Journal of Infectious Diseases and Medical Microbiology. 2005; 16(4):245- 248.

12. Falcone EL, Petts JR, Fasano MB, Ford B, Nauseef WM, Farela Neves J, et al. Methylotroph infections and chronic granulomatous disease. Emerging Infectious Diseases. 2016; 22(3):404-409.

13. Greenberg DE, Shoffner AR, Zelazny AM, Fenster ME, Zarember KA, Stock F, et al. Recurrent Granulibacter Bethesdensis infections and chronic granulomatous disease. Emerging Infectious Diseases 2010; 16(9):1341-1348.

14. López FC, Luna FF de, Delgado MC, Ibarra de la Rosa I, Valdezate S, Saez Nieto JA, et al. Granulibacter bethesdensis isolated in a child patient with chronic granulomatous disease. Journal of Infection. 2008; 57(3):275-277.

15. Batty M, Zelazny AM, Church JA, Chase JM, Holland SM, Greenberg DE, et al. Acidomonas methanolicaassociated necrotizing lymphadenitis in a patient with chronic granulomatous disease. Journal of Clinical Immunology. 2012;32(6):1193-1196.

16. Lai CC, Cheng A, Liu WL, Che-Kim Tan, Yu-Tsung Huang, Kuei-Pin Chung, et al. Infections caused by unusual Methylobacterium species. Journal of Clinical Microbiology. 2011;49(9):3329-3331.

17. Cuellar CM, Arbo A, Condino Netto A, Donatto J, Muscara MN, Carneiro Sampaio MMS, et al. Doenca Granulomatosa Crónica (DGC) por Deficiencia de p47phox em duas Irmas. 2th Panamerican Congress of Pediatric Allergy and Immunology and 5th Brazilian Congress of pediatrie Allergy and Immunology, 1995 mar 16-22. San Pablo, Brasil; 1995.

18. Mouy R, Fischer A, Vilmer E, Seger R, Griscelli C. Incidence, severity, and prevention of infections in chronic granulomatous disease. J Pediatr. 1989; 114(4):555-60.

19. Kuhns DB, Alvord WG, Heller T, Feld JJ, Pike KM, Marciano BE, et al. Residual NADPH oxidase and survival in chronic granulomatous disease. N Engl J Med. 2010; 363(27):2600-10.

20. Jones LB, McGrogan P, Flood TJ, Gennery AR, Morton L, Thrasher A, et al. Special article: chronic granulomatous disease in the United Kingdom and Ireland: a comprehensive national patient-based registry. Clin Exp Immunol. 2008; 152(2):211-8. 
21. Marciano BE, Spalding C, Fitzgerald A, Mann D, Brown T, Osgood S, Yockey L, et al. Common severe infections in chronic granulomatous disease. Clin Infect Dis. 2015; 60(8):1176-83.

22. Arbo A, Martínez de Cuellar C, Moreno Azorero R. A propósito del primer caso de Enfermedad Granulomatosa Crónica descrita en Paraguay. Ped. (Asunción)1992; 19(2):6-10.

23. Roos D, Kuhns DB, Maddalena A, Bustamante J, Kannengiesser C, de Boer $\mathrm{M}$, et al. Hematologically important mutations: The autosomal recessive forms of chronic granulomatous disease (second update). Blood Cells Mol Dis 2010; 44:291-299.

24. Roos D, Kuhns DB, Maddalena A, Roesler J, Lopez JA, Ariga T, et al. Hematologically important mutations: $X-$ linked chronic granulomatous disease (third update).
Blood Cells Mol Dis 2010; 45:246-265.

25. Winkelstein JA, Marino MC, Johnston RB Jr., Boyle J, Curnutte J, Gallin JI, et al. Chronic granulomatous disease: Report on a national registry of 368 patients. Medicine 2000;79:155-169.

26. Song E, Jaishankar GB, Saleh H, Jithpratuck W, Sahni R, Krishnaswamy G. Chronic granulomatous disease: A review of the infectious and in?ammatory complications. Clin Mol Allergy 2011; 9:10.

27. Borges de Oliveira-Junior J, Bengala Zurro N, Prando C, Cabral-Marques O, Soeiro Pereira PV, Schimke LF, Klaver S, et al. Clinical and Genotypic Spectrum of Chronic Granulomatous Disease in 71 Latin American Patients: First Report From the LASID Registry. Pediatr Blood Cancer. 2015 Dec; 62(12):2101-7. 